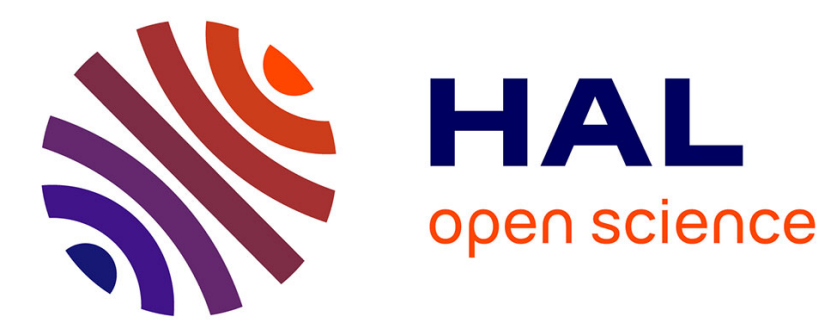

\title{
Sur la mesure de la constante de J'émanation du radium Pierre Curie
}

\section{To cite this version:}

Pierre Curie. Sur la mesure de la constante de J'émanation du radium. Radium (Paris), 1910, 7 (2), pp.33-38. 10.1051/radium:019100070203300 . jpa-00242390

\section{HAL Id: jpa-00242390 https://hal.science/jpa-00242390}

Submitted on 1 Jan 1910

HAL is a multi-disciplinary open access archive for the deposit and dissemination of scientific research documents, whether they are published or not. The documents may come from teaching and research institutions in France or abroad, or from public or private research centers.
L'archive ouverte pluridisciplinaire HAL, est destinée au dépôt et à la diffusion de documents scientifiques de niveau recherche, publiés ou non, émanant des établissements d'enseignement et de recherche français ou étrangers, des laboratoires publics ou privés. 


\title{
MÉMIOIRES ORIGINAUX
}

\section{Sur la mesure de la constante de l'émanation du radium}

\author{
Par $M^{\text {me }}$ P. CURIE
}

[ Tincultr des Sciences dr. Paris].

La vitene de destruction de lémantion du radium a dijat éte l'objet de plu-ieur- déterminations. Ioici les valeurs qui ont été indiquées pour la période T de diminution de moilic 1.

\begin{tabular}{|c|c|c|c|}
\hline P. Curie. . . & & $\overline{7.99}$ & ours \\
\hline Rutherford et Soddy. & 1) & $5.7 !$ & ॥ \\
\hline Bumstead et Wheeler. & 1) & 5.88 & 1) \\
\hline Sackur. . . . . . & ) & 5.86 & 1) \\
\hline Rümelin. . . . . . & ) & 5.75 & ) \\
\hline
\end{tabular}

P. Curie mesurait à des intervalles de temps convcnables l̈ionisation produite dans un appareil de mesures par une certaine quantité d'émanation contenue en vase clos, et agissant, soit par son rayomement total, soit par le rajonnement pénétrant. MII. Rutherford et Soddy conservaient l'émanation dans une éprouvette graduée et en prenaient de temps en t'mps un volume déterminé qu’ils introduisaient dans l'appareil de mesure; cette même méthode a été utilisée par M. Sackur. M. Rïmelin partageait une certaine quantité d'émanation en deux portions dans un rapport connu, el mesurait, après des intervalles de temps différents, l'activité de chaque portion ; lémanation était transportée dans lappareil de mesure par un courant de rapeur d'cau qui l'entraînail dans un gazomètre à eau, d'où clle étail aspiréc dans la chambre d'ionisation. Les nombres obtenus pour la période T par les différents expérimentateurs laissent subsister une incertitude de quelyue- pour lo1) sur la valeur de cette période. et il paraissait desirable de connaile plus exactement une constanle anssi impor-

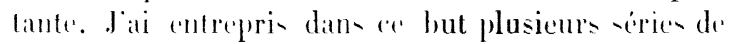
me-ures, dont les résultats sont indiques dans ce mémoire.

La méthode utilise etait celle de P. Curie qui consiste à observer lantivité de lémanation nntermée

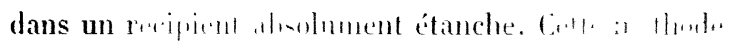

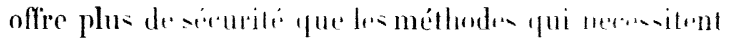

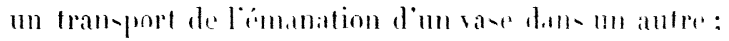

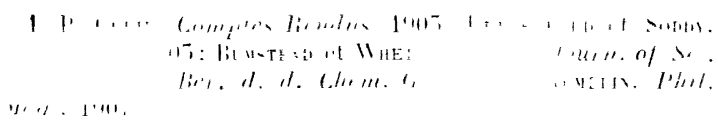

T. 7. les canses dierreur qui résultent du transport sonl angmentés aree le dispositif de II. Rimmelin far lusage du gasomètre à eau; l'émanation alıant une solubilité appréciable (coefficient de solubilité environ $0, \bar{y}$ à la t(emperature ordinaire), il doit se produire une perle d'imanation qui n'est pas négligeahle pour des expériences précises.

Le rayonnement mesuré était, soit le rajonnement pénétrant, soit le rayonnement total. L'appareil utilisé pour la mesure du rajonnement pénétrant est représenté dans la figure 1; il est très analogue à

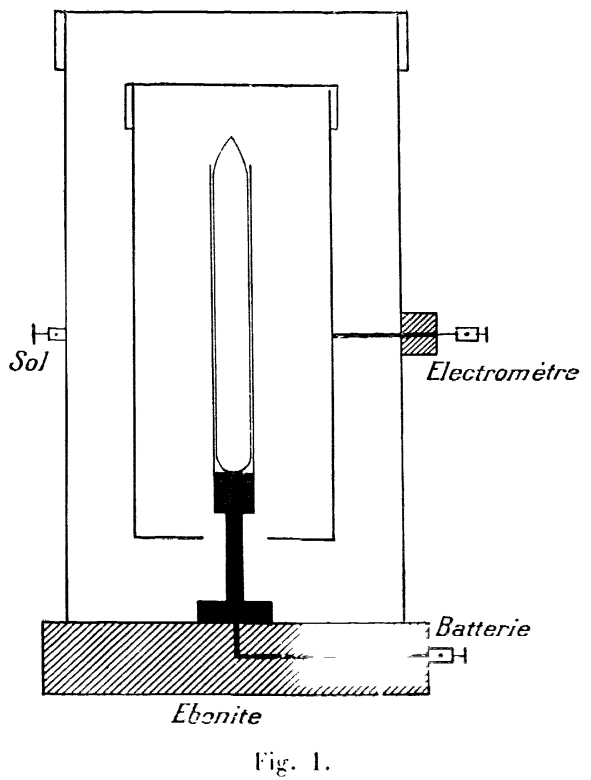

lappareil primitif de P'. Curie. L'amanalion al antenue dans un tube de serre serlle. place a linterintr diun tule daluminium minte: re dernier tormen

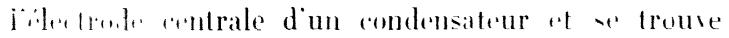

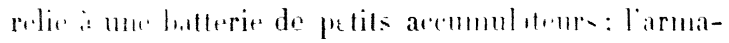

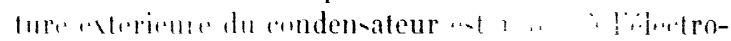

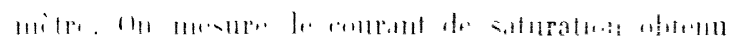

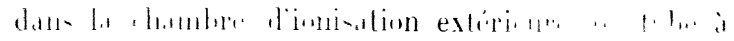
imantion: la mithende at dite $m$ in trutes erierieures. 
Dans d autres expériences, on mesurait l'ionisation produite à lintérieur d’un récipient par l'émanation qui ýtait contenue. Cette méthode est celle des électrodes intérieures. Lappareil utilisé est un condensiteur à qa de modèle courant (lig. 2). La boìte de

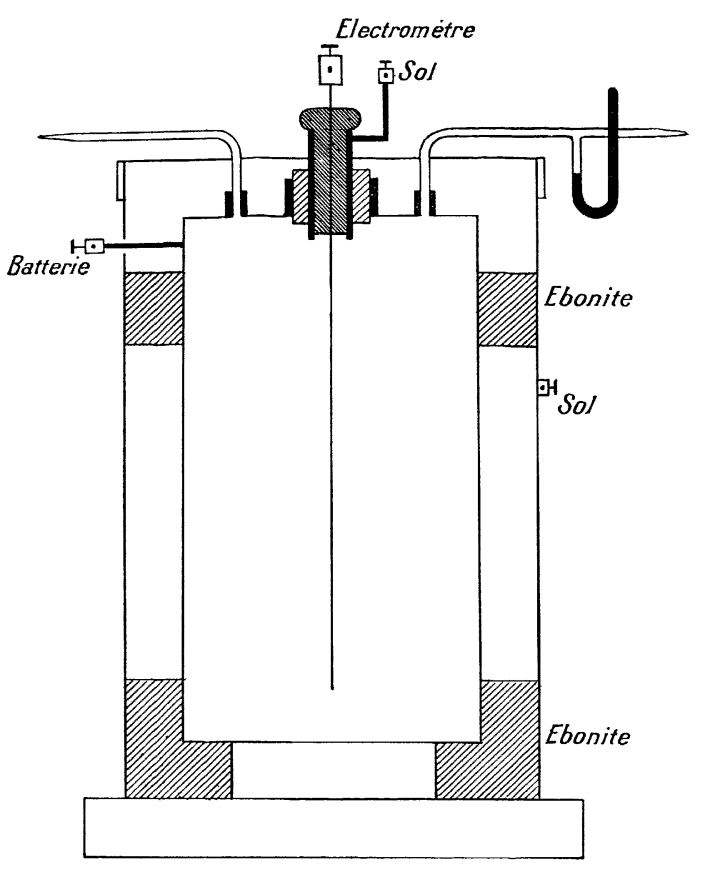

Fig. 2.

ce condensateur est munie de deux tubes de dégagement étroits, dont l'un se termine par un manomètre tronqué de très petit volume, tandis que l'autre sert de tube de communication. L'opération préliminaire consiste à faire le vide dans le condensateur et à fermer le tube de communication à la lampe. Ón laisse ensuite l'appareil en observation pendant quelques jours pour véritier qu il est étanche. On aspire alors dans le condensateur une quantité convenable d'émanation contenue dans de l'air sec, on finit de remplir avec de l'air sec jusqu'à ce que la pression devienne sensiblement égale à la pression atmosphérique et l'on ferme à la lampe le tube qui a servi pour laspiration. Lappareil est alors prèt pour une série de minsuren.

Les mentures bitient effertuce au moven diun

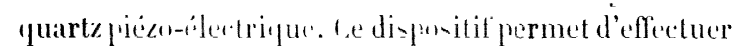
de ey rin..... de longue dure sur un ravonnement dintensite decroirsante. toutes lesmesures titant absolument comparahles "ltre ellos sans que l'on ait ì - inquiéter de la rmilsilite de lélectromitre et de

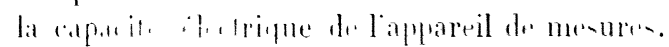

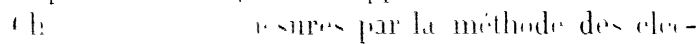

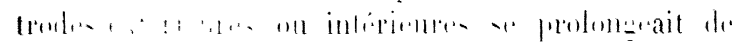

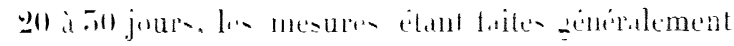
une ou deux tui- far jour. Le maximum de lintensile: du courant est atteint $\overline{5}$ heures après le moment où l'émanation a été aspiréc dans le condersateur; toutefois la loi exponentielle pour la diminution de l'intensité ne semble parfaitement établie que 2 ' heures après l'aspiration. J'ai cru conslater en particulier que dans mes expériences à électrodes intérieures, le courant, après a voir atteint le maximum, décroìt de plus en plus vite entre $t=5$ heures et $\boldsymbol{t}=\mathbf{2 0}$ heures, le temps étant compté depuis l'instant où l'émanation a été introduite dans l'appareil ; mais à partir de $t=20$ heures, la vitesse de baisse devient un peu moins grande, et prend un régime constant qui se maintient ensuite pendant plus de 20 jours. Si donc on construit la courbe qui représente le logarithme de l'intensité en fonction du temps, cette courbe présente un point d'inflexion entre $t=4$ heures et $t=20$ heures. Ce point d'inflexion, dont l'existence n'est pas prévue par la théorie, est peu marqué, et il est difficile d'ètre tout à fait affirmatif à son sujet, cependant je l'ai observé plusieurs fois; la pente moyenne de la courbe entre 4 heures et 20 heures correspondait à une diminution du logarithme décimal par heure $a=0.00345$, tandis que la pente régulière qui représente la décroissance de l'émanation entre 1 jour et 50 jours correspond à la valeur $a=0.00326$. La netteté du point d'inflexion pourrait dépendre des dimensions de la chambre d'ionisation. Ces dimensions étaient les suivantes :

Diamètre intérieur du cylindre : $6,7 \mathrm{~cm}$.

Hauteur intérieure du cylindre ; 12,5 cm.

Volume du condensateur : $440 \mathrm{~cm}^{3}$ environ.

L'électrode intérieure de $5 \mathrm{~mm}$ de diamètre a une longueur de $11,5 \mathrm{~cm}$.

Dans toutes les expériences le courant de saturation était toujours atteint; la différence de potentiel établie entre les deux armatures du condensateur de mesures élait de 800 volts.

Dans les expériences faites par la méthode des élcctrodes intérieures, la masse de gaz soumise à l'eflet ionisant restait constante; aucune correction n'était done nécessairc pour tenir compte de la rariation de la pression et de la température de l'air ambiant. Yais dans lus expériences faites avec l'appareil à électrodes extéricures, la chambre d'ionisation n'était pas close de manière à être étanche. L’ionisation produite dans l'air contenu dans cette chambre par les rajons pénétrants qui sortent du tube à émanation croil avec la densité de l'air soumis à l'action des rilsons: elle pourrait itre considérée comme proportionnelle à cette dewiti. sil élait certain que le pouroir pénétrant de tou la rayons utiliece at suftisamment srand. 11 m aurait in ce cas :

$$
\frac{i .}{i}=\frac{l_{1 \prime}}{d}=\frac{l^{\prime \prime}}{l^{\prime}} \frac{1+\% l}{1+\% t}
$$

en designant par $i$ le courant mesuré à la pression $\mu^{\prime}$ 
et la température $t$. et jar $i$ le courant rapporté à une pression $l$, et une temperature $t_{n}$. Lil correction $\Xi$ qui est à ajouter à $i$ pour obtenir $i_{0}$ est alors donnée approvimatirement par la formule

$$
\left.\varepsilon=i: 0.0015(760-1)+0.0057 \cdot t-t_{0}\right)
$$

où $p$ est mesuré en $\mathrm{mm}$ de mercure: la valeur de $\Xi$. géníralement faible, peut cependant atteindre jusqu'à 2 pour 100.

Cette correction est probablement un peu trop lorte, car il ent raisemblable que parmi Ies rayons primaires el secondaires il ! en a de relittivement absorbables: cejerndant leyperience montre que la correction change trës jeu l'arpect des courbes obtenues, et qu'clle n'est pas susceptible de modifier les résultats.

Une autre correction est à considérer; c'est celle qui résulte de la radioactivité propre de l'appareil de mesures. Celle-ci était toujours très faible au début de l'expérience, par exemple 0,1 pour $100 \mathrm{du}$ courant à mesurer; elle augmentait d'importance à mesure que le courant dì à l'émanation allait en diminuant, et elle pouvait atteindre alors ò pour 100 de la valeur à mesurer. Lne série de mesures portait sur 50 jours environ. Quind on emploie le procédé des électrodes exlérieures, on peut prolonger la durée de l'expérience en employant une grande quantité d'ímanation, et en réduisant au début lintensité do ravonnement au moven d'un écran a'n plomb épais yui enveloppe le tule ntil et que lion enlive uprie 2) jours. On comblate loutefois qu il n! " par intipit is

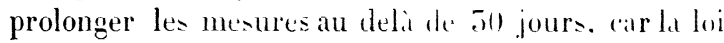
exponentielle simple se trous altére at partir de

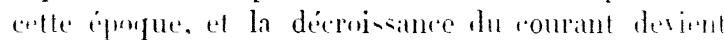

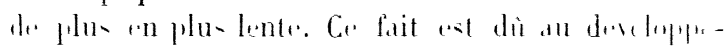

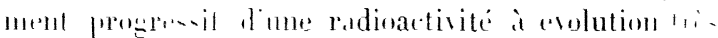
linte différente de colle qui ne demande qu. 5 hreure pour atteindre un épuilibre de régime arce linumation du radium: il : a lormation de radium It suivie de production de radium E qui donne lieu i une émission de råons pénétrants. Il es important de sassurer qu ì la fin de lintersalle de temps utilisé. le rayomement du radium $\mathrm{E}$ ne peut contribuer dunc inanière appréciable à produire le courant danl'appareil de mesures. En effet i ce rayommement commencait à se fatire sentir. la loi de décroissance
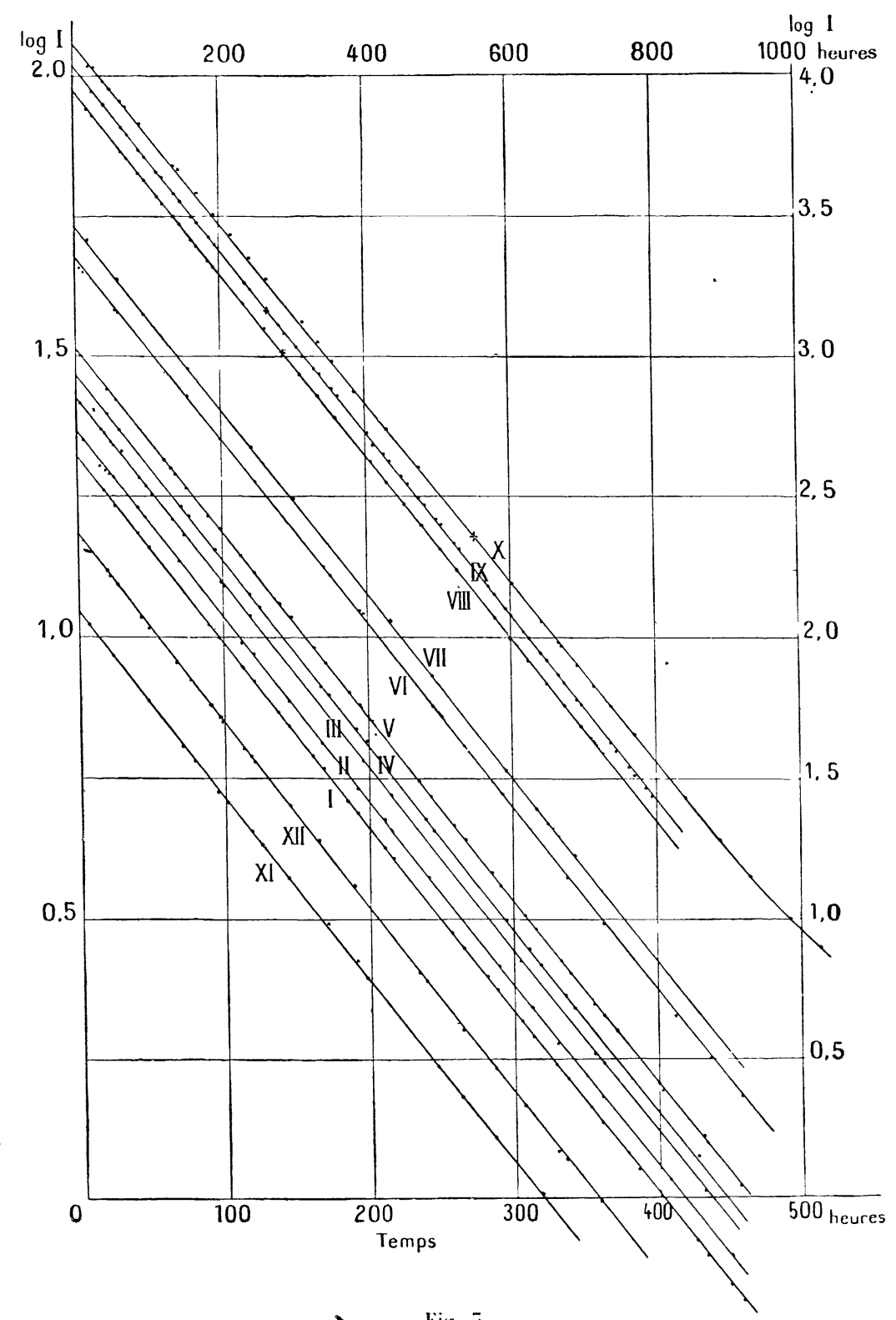

Firg. .7.

observie serait un peu plus lente que celle qui catractérise lémanation. la détormation frousut a-

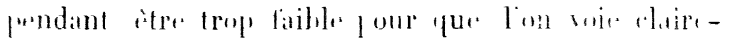

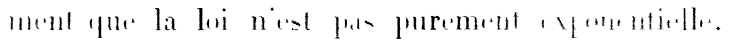

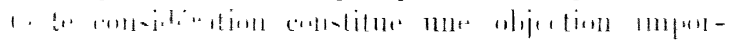
tante contere lemplui de methole qui com-i-lent is

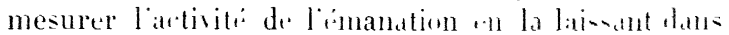

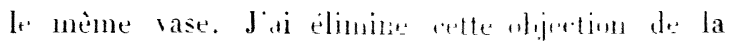


manière suivante. (luand la sírie des mesures est termince, on chasse l'émanation du rase qui la contient, on attend 2o' heures pour liisser s’éteindre la radioactivití induite à ćvolution rapide, et l'on mesure alors le ravo nement propre de l'aplareil. Si l'on opère arec des électrodes intéricures, le vase est simplement le condensateur de mesures; si l'on opìre avec des électrodes extérieures, ce vase est le tube qui contenait l’émanation et qui, après avoir été ridé. doit ètre remis en place pour la vérification à faire après 2' $\mathbf{y}$ heures. Dans les deux cas on constate que lactivité observée ainsi après une expérience de Q̇ jours est très faible et qu'elle ne saurait modifier les résultats obtenus.

Chaque sírie de mesures a été représentée par une courbe, en portant en obscisses le temps mesuré en heures, et en ordonnées le logarilhme décimal du nombre qui mesure le courant en unités arbitraires.

Les courbes ainsi obtenues sont reproduites dans la figure 5. Ce sont très exactement des droites dont le coefficient angulaire $a$ (diminution du logarithme décimal de l’intensité par heure) est indiqué dans le table:ıu qui suit :

Tableau I : Électrodes intérieures.

\begin{tabular}{|c|c|c|}
\hline & Temps dolservation. & $a$. \\
\hline $\begin{array}{l}\text { I } \\
\text { II } \\
\text { III } \\
\text { II } \\
\text { I }\end{array}$ & $\begin{array}{l}470 \text { heures } \\
150- \\
400- \\
450- \\
580-\end{array}$ & $\left.\begin{array}{l}0.005279 \\
0.005270 \\
0.005265 \\
0.005280 \\
0.005258\end{array}\right\}$, valcur moyenne \\
\hline
\end{tabular}

L'échelle relative à ces droites est indiquée sur la figure en bas el ì gatuche.

Tableau II : Électrodes extérieures.

\begin{tabular}{|c|c|c|}
\hline & Temps dobservation. & $a$. \\
\hline $\begin{array}{l}\text { III } \\
\text { III } \\
\text { VIII } \\
\text { II } \\
\text { II }\end{array}$ & $\begin{array}{r}460 \text { heures } \\
360 \quad- \\
700- \\
800- \\
1040-\end{array}$ & 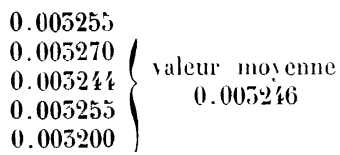 \\
\hline
\end{tabular}

Les droites VI et III sont construites à la mime échelle que celles du tableau précédent. Les droiles VIII, II. I représentent des expériences de durée beaucoup Ilu- erande ct sont construites it une échelle moitié de la précidente, indiquée sur la fizure en haut et à droite. La concentration initial. de l'imanation dans lo tube diat en ce ca plu- :rande. mais löntensiti du rayonnement au dehors che rit riduite par lemploi

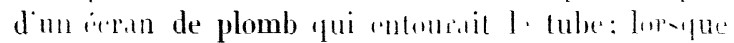

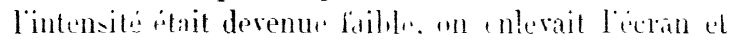
lon contimmit la mesures du- h meme dplareil. Le raccord des deux parties de la droite obtenues avec et sans écran est indiqué sur la figure par une croix, et l'on voit qu'il n'y a en ce point aucun changement de dircction. L'altération de la droite $\mathrm{I}$ à partir de 800 heures est due à l'apparition de la radioactivité induite à érolution lente.

Dans les expériences du tableau 2 la concentration initiale de l'émanation était considérablement plus grande que dans les expériences du tableau 1, le rapport ćtant de l'ordre de $10^{\circ}$. On remarque aussi qu'au cours d'une expérience isolée la concentration peut diminuer dans un rapport de 1003 environ sans que la loi exponentielle se trouve en défaut. De plus le coefficient $a$ est sensiblement le même dans toutes les expériences, quelle que soit la concentration initiale dans les limites indiquées. Il en résulte que la loi de destruction de l'émanation est indépendante de la concentration de celle-ci dans de très larges limites.

Ce fait est encore confirmé par les expériences faites avec de l'émanation très concentréc (quantité maximum d'émanation pouvant itre fournic par un décigramme de radium et contenue dans un petit tube de volume $0,1 \mathrm{~mm}^{3}$ environ). La concentration initiale en ce cas était $2 \times 10^{11}$ fois plus grande que dans les expériences avec électrodes intérieures, et eependant la loi de destruction ne paraît pas altérée. La droite XI représente une de ces expériences avec $a=0.00525$.

La droite XII représente le résultat d'une série de mesures obtenues dans un appareil à électrodes intérieures avec l'émanation restante d'une expérience faite précédemment dans l'appareil à électrodes extéricures. La loi de destruction est toujours sensiblement la même $(a=0.00550)$. A la fin de cette séric l'émanation rtait séparée du radium depuis 5 mois.

On voit d'après cela que la constante de l'émanation est susceptible d'être déterminće avec une grande précision par les deux méthodes employées. La mélhode des électrodes intérieures donne pour $a$ des valeurs plus concordantes et, en moyenne, légèrement supérieures à celles fournies par l'autre méthode. (Écart des movennes inférieur à 1 pour 100). La méthode des électrodes intérieures, dans laquelle le ravonnement de l'émanation intervicnt directement à cóté de celui de la radioactivité induite, semble avoir de ce chef un avantage sur celle des électrodes extérieures, dans laquelle le rayonnement de la radioactivité induite intervient seul; toutefois cette dernièrc méthode présente en revanche plus de sécurité p.rce qu il est certain qu'aucune fuite d’émanation ne peut avoir lieu.

Lit héorie montre d'ailleurs, que quelques heures dipis lointroduction de l'émantition dans le récipient, 1. rasomnement total et le rạonnement pénétrant sont tous les deux très exactement proportionnels à la quantité d'émanatioa, et décroissent suirant lit 
mime loi que celle-ci. pendint löintervalle de lemputilizé dans le- expériences.

Jai remarqui pue les re-altats mbtenus are desélectrodes esterieures sont trés inlluencés par l'ital d'humidite de l'air qui contient l'́manation. si. en particulier, celle-ci est rizoureusement desséchíe, les résultats sont très irréguliers, à tel point que l'erpérience ne peut pas ètre utilisée pour la détermination de la constante. Ca phénominc explique par les conditions de dépòt de la radivactivité induite dans le tube qui contient lémanation. Ce dépòt se forme dans le gaz et se dépose sur les parois du tube par un phénomène de diffusion; celui-ci est fortement influencé par les variations de lis température ambiante qui donnent lieu à la production de remous dans la masse gazeuse. Tout changement de distribution du dépòt actif peut se traduire par une variation de lintensité d'ionisation dans l'appareil qui utilise les rayons. Un peut régulariser le phénomène par deux procédés : $1^{\circ}$ en utilisant un tube très étroit, dans lequel le dépòt actif atteint très facilement la paroi et ne séjourne pas dans le gaz; une telle expérience est représentée par lı droite VII; $2^{\circ}$ en utilisant de l'air trìs humide dans lequel le dépòt actif subit l'action de la pesanteur et atteint rapidement la paroi inféricure; la droite VI représente une expérience faite dans ces conditions.

On peut conclure qu'en adoptant la valeur $a=0.0(0) 6$, on obtient la constante de l'émanation du radium avec une erreur inférieure à 1 pour 100 . D'après cette valeur de $a$ on trouve :

$$
\begin{aligned}
& \lambda=2.08510^{-6} \frac{1}{\mathrm{sec}}=0.007 \mathrm{j} 1 \frac{\mathrm{l}}{\text { heure }} \\
& \Theta=4.79610^{3} \text { sec. }=155.2 \text { heures } \\
& \mathrm{T} \quad 3.52410^{\circ} \text { sec. }=92.5 \mathrm{~h}^{\prime}=5.85 \text { jours. }
\end{aligned}
$$

Pour controler les résultats des expériences qui viennent d'itre décrites, une autre méthode de détermination de la constante $\lambda$ a ingalement été essayée. On étudiait l'accroissement d'activité an fonction du temps pour un sel ritdifère qui, après avoil ité ramlené ì -on alctivite minimum, a bli antermei en lulue scelle. Pour priver le sel de limanittion el dı dépòt actit. on le dissout et l'on chasse l'émanatime en laissant sacromplir lit destruction du depoit antif areumuli. En-uite la dissolution at rapid.ment irapure it see. it le et el introduit dam une ampoule de verre itroite. In me-1tre le ranmusment pénitrant de cette ampoule atu mosen diun dispesitil "eperimental en tout point -emblahle it celui qui sert prour la mesure de la decroisente de linted-

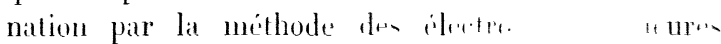
(fiz. 1). Lintem-iti du rasonnement. diand trifaible, croit proseresivement et tend vers une limite déterminée; cette intensité est à chaque instant proportionnelle à la radioactivité induite formíe dans le - 4 . et celle-ci est elle-mème proportionnelle à li yuantité démanation q présente dins le sel apris le temps $t$, à condition que ce temps soit suprírieur à quelques heures, et qu'un équilibre de régime 'nutre l'émanation et la radioactiviti induite soit établi. La quantité d'émanation $q$ tend vers sa valeur limite $q$. suirant la loi

$$
q=q_{*}\left(1-e^{-i \prime}\right)
$$

ò̀ $\lambda$ est la con-tante de l'émanation.

$$
\text { On adone } \quad \frac{q_{x}-q}{q_{x}}=e^{-i . l}
$$

L'excès de l'aclivilí limite sur l'activiti actuella décroît donc suisant la loi de destruction de l'ćmanation. Si l'on porte en abscisses le temps, et cn ordonnées le logarithme de la diflérence $\left(q_{x}-q\right)$, les points obtenus doivent se placer sur une ligne droite dont linclinaison permet de calculer la constante $\lambda$.

Plusicurs expériences faites dans ce but ont domné des résultats tout ì fait conformes à la théoric. La diminution du logarithme décimal de $\left(q_{x}-q\right)$ par heure est très exactement la mème que celle observée sur les droites de la figure $\overline{7}$. Les nombres obtenus dans trois expériences consécutives sont :

$$
0.00 \pi 17 \quad 0.00526 \quad 0.00322
$$

Liune des expériences est représentée dans la ligure 4 .

Cependant cette meihode ne semble pas - urepplible

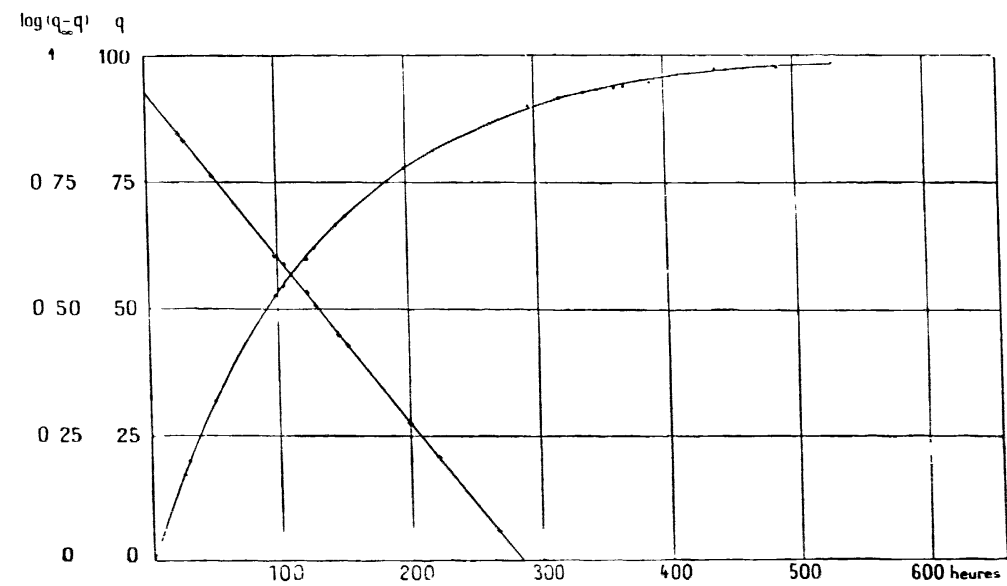

Fig. 4

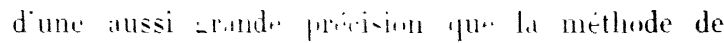

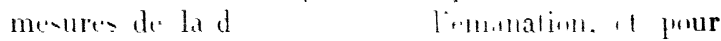


wtte rairnn le- nombres obtenus niont pas ité utilisés pour la détermination de la constante. Lerreur commise sur lintensité limite se fait sentir sur chacune des différences $q .-q$; les courbes obtenues ne sont bien régulières yue sur un intervalle de 8 ì 10 jours environ.

I.cs valeurs des fonctions $e^{-i l t}$ et $1-e^{-i t}$ pour l'éma- nation du radium ont été indiquées par II. Kolowral' pour diverses valeurs de $t$, dans des tables construites ì cet effet. La valeur de $\lambda$ qui a servi pour le calcul est celle yui a íté déduite des déterminations décrites ci-dessus.

[Reçu le 14 Février 1910.] 